\title{
Granulocyte-Colony stimulating factor induced early psoriasis - a case report and literature review
}

\section{Manandhar Upasana, Wang Yue Yin, Wu Jian Bo, Song Ji Quan}

\author{
Department of Dermatology and Venereology at Zhongnan Hospital of Wuhan University, Wuhan City, Hubei Province, \\ China
}

Corresponding author: Prof. Song Ji Quan, E-mail: songjiq@126.com

\begin{abstract}
Human Recombinant Granulocyte colony-stimulating factor is a hematopoietic growth factor; most commonly known to be used for chemotherapy induced neutropenia for the mobilization of peripheral blood stem cells. A case of 43 years old lady, post radical mastectomy for breast cancer and one cycle of chemotherapy was encountered with the development of massive psoriasis like eruptions all over the body including the scalp with adherent silvery scales after 10 days of administration of granulocyte colony stimulating factor with failure of remission even after months of treatment. Clinical signs of psoriasis were seen positive. Munro micro abscess was also noted in the Biopsy. Patient was treated as adverse drug reaction with immediate cessation of G-CSF administration. The rash improved significantly with no new appearances. However, during follow up, island of patches persisted on certain areas, and soon after there was second round of massive eruption without the drug administration. It is very unusual for a drug reaction to not heal after the cessation of the drug. Moreover, since there was second round of sudden massive eruption without the drug, it was clearer that it was not a drug reaction. Rather, the clinical signs and the biopsy were suggestive of early psoriatic presentation.
\end{abstract}

Key words: Granulocyte colony stimulating factor; G-CSF; Psoriasis; Adverse drug reaction; Neutropenia

\section{INTRODUCTION}

Granulocyte colony-stimulating factor (G-CSF) is a hematopoietic growth factor (HGF) that stimulates the production and function of granulocytic cells mostly neutrophils [1]. The incorporation of human recombinant G-CSF as a therapeutic tool has allowed an exogenous stimulation of hematopoietic precursors in order to increase the number of circulating neutrophils [2]. These agents are used frequently to promote leukocyte recovery and reverse resultant myelosuppression after high dose chemotherapy. They have made a substantial clinical impact especially in the management of cancer patients. As every coin has two sides, there have been reports of various adverse effects both cutaneous and systemic. Therefore, we here have a case of initial drug eruption induced by G-CSF administration in a patient with breast cancer, which was later recognized to be early psoriasis.

\section{CASE REPORT}

Patient, female, 43 years old, post radical mastectomy and one cycle of chemotherapy presented to the outpatient department of our hospital with extensive erythema, maculopapular rashes and desquamation for two weeks. She had undergone one cycle of chemotherapy (docetaxel 140mg, cyclophosphamide $1140 \mathrm{mgdl}$, q2 $1 \mathrm{~d}$ ) according to the TC regimen with necessary adjuvant therapy. On the tenth day after chemotherapy, the patient developed bone marrow suppression for which the patient was given recombinant Human granulocyte colonystimulating factor (G-CSF). On the same day of the administration of G-CSF, the patient developed a

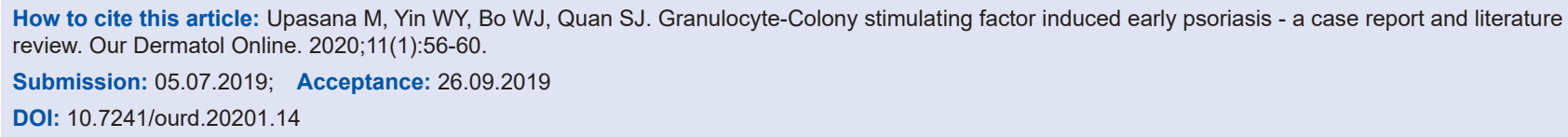




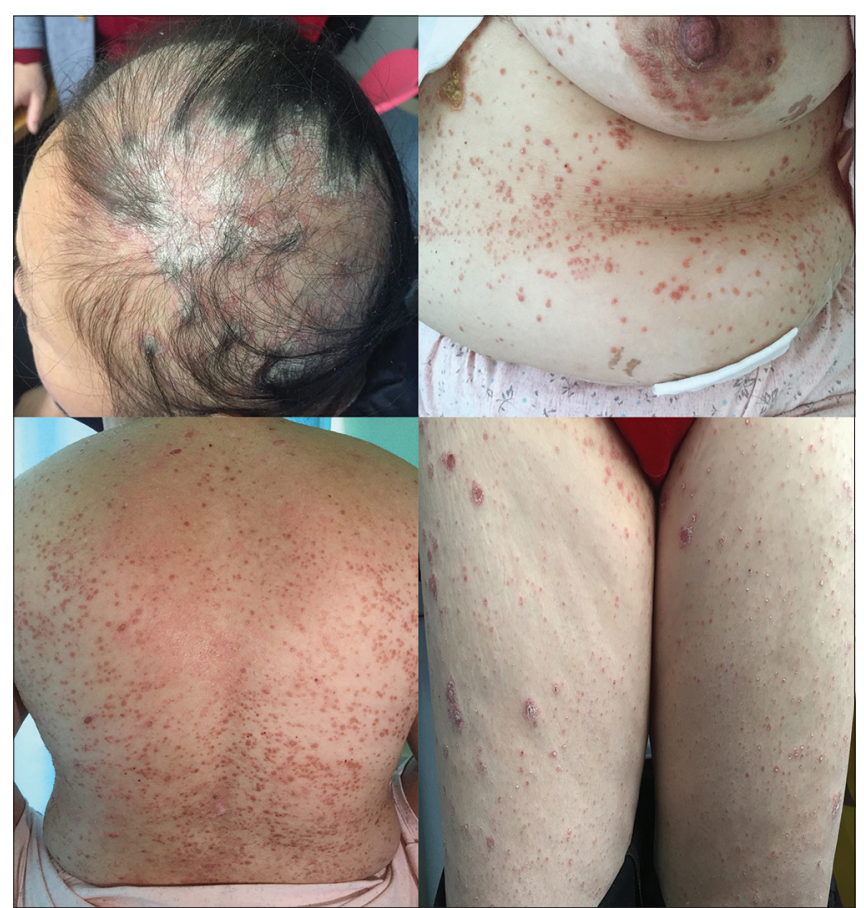

Figure 1: Erythematous maculopapular rashes and patches with silvery white scales over the scalp, abdomen, back and thighs at presentation.

red rash on the right side of her armpit, which spread to the chest and buttocks, and gradually spread over the whole body including the scalp. Numerous small miliary rashes to large erythematous patches covered with adherent silvery white scales were noted (Fig. 1) with no pruritus or pain. Her lesions were extensive and were increasing rapidly. Newly appeared fresh red rashes as well as faded brown maculopapular rashes, covered with silvery white scales could be observed on the trunk and limbs with positive Film phenomenon and dot bleeding sign (Auspitz sign). Thick white scaly patches were seen on the scalp with fine demarcation extending outside the hairline with scarce hair (Fig.1). The patient had no other significant past medical history and no systemic abnormalities were detected. Biopsy showed hyperkeratosis of the epidermis, parakeratosis, and neutrophil infiltration in the stratum corneum with Munro micro abscess formation, mild spongiotic changes in the epidermis and mild perivascular infiltration in the superficial dermis (Fig. 2). After the comprehensive analysis of patient's medical history and ongoing chemotherapy it was termed as G-CSF induced drug eruption.

She was then treated with anti-allergic drugs in combination with topical therapies, which showed significant improvement of her rashes with no new eruptions. On follow up after two months, the

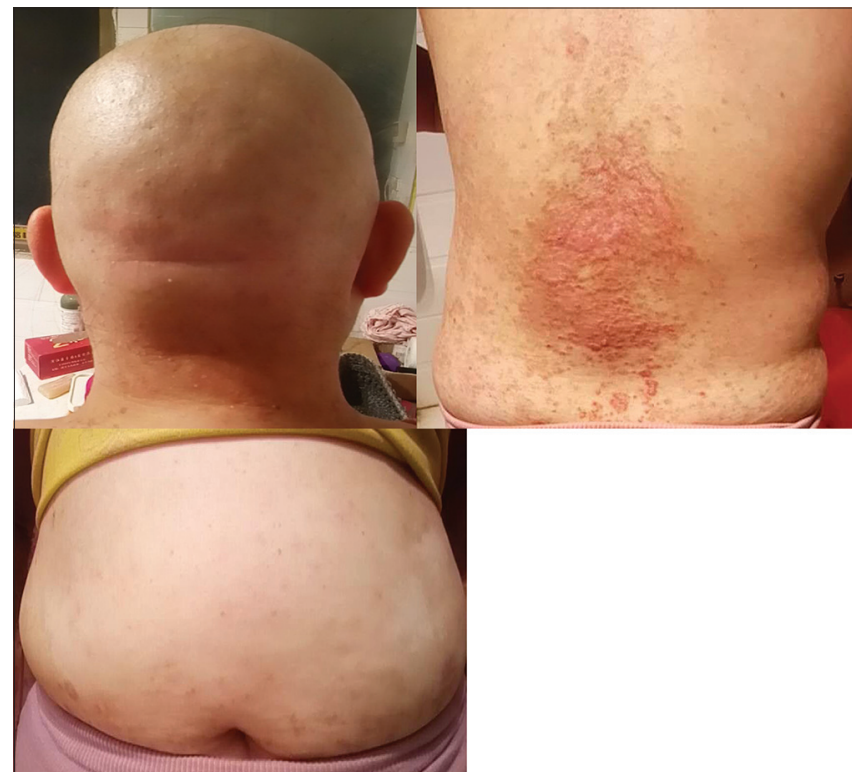

Figure 2: Complete remission of lesions over the scalp and abdomen. Residual maculopapular rashes and island of patches over the back after 2 months during follow up.

rashes on her scalp, abdomen and extremities had completely cleared but islands of patches persisted on her back with large erythematous maculopapular rashes without scales (Fig. 3). Therefore, the patient was under simultaneous treatment for her cancer and the rashes. But again after 4 months of initial eruption she had second round of sudden extensive eruption all over her body (Fig. 4). The lesions varied from small red maculopapular rashes to large scaly patches with adherent silvery scales similar to the first episode of eruption. Auspitz sign and film phenomenon were seen positive. Patient however refused to do another biopsy and insisted on supportive management.

Since the second time had no any aggravating factors and the clinical manifestation was favorable for psoriasis. Finally, after overall analysis of the case, the final diagnosis was made as psoriasis induced upon the administration of G-CSF 4 months ago, the initial drug eruption being the early psoriatic presentation.

\section{DISCUSSION}

Psoriasis is one of the most common chronic inflammatory skin disorder predominantly characterized by accumulation of Thl-type $\mathrm{T}$ cells and neutrophils, rigorous epidermal proliferation and differentiation, and enhanced epidermal production 


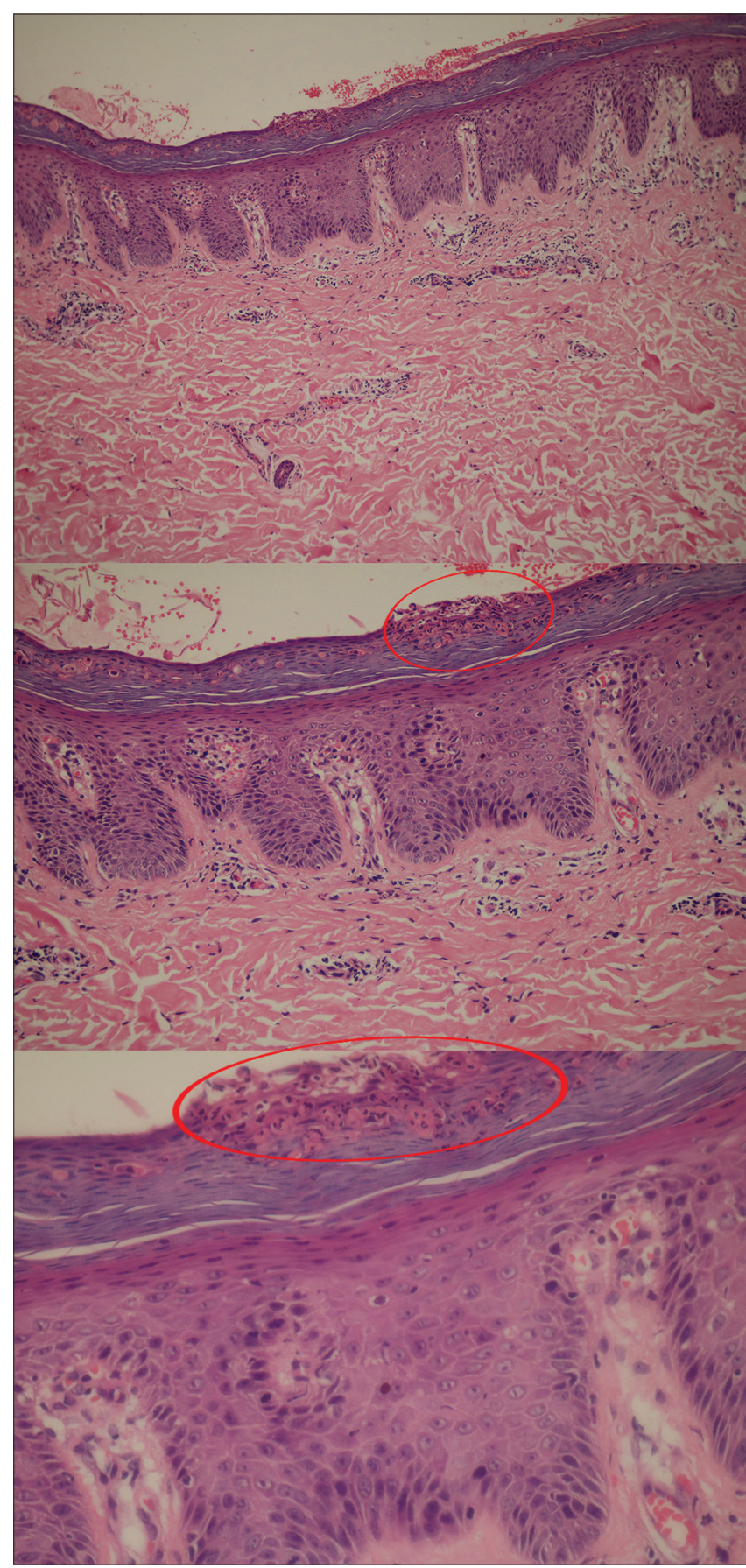

Figure 3: Biopsy (Hematoxylin and Eosin stain, 20x, 40x and 100x) showing hyperkeratosis of the epidermis, parakeratosis, and neutrophil infiltration in the stratum corneum with Munro microabscess formation(circled), mild spongiotic changes in the epidermis and mild perivascular infiltration in the superficial dermis.

of antimicrobial peptides [3]. Granulocyte colony stimulating factor (G-CSF) is a cytokine that is commercially available as a result of recombinant DNA technology $[4,5]$. Human recombinant G-CSF was used for the treatment of neutropenia induced by chemotherapy in the presented case. In this case the first lesions were seen on the 10th day of administration

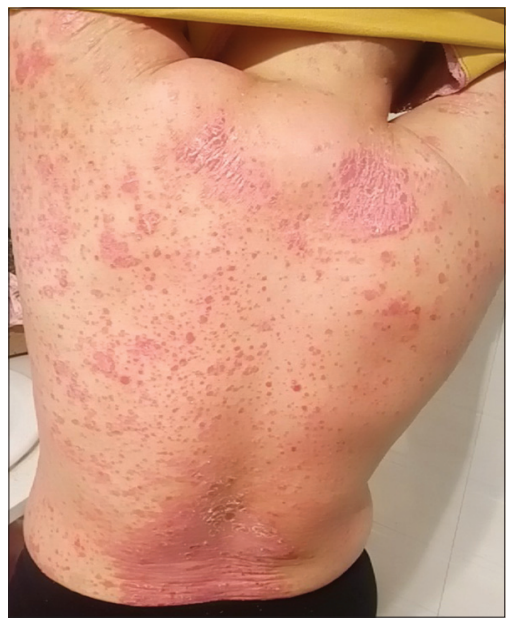

Figure 4: Second round of extensive eruption.

of G-CSF after first cycle of chemotherapy. Within this short period of time, the patient had developed erythematous macules and patches with silvery white scales all over the body including the scalp, with positive film phenomenon and Auspitz sign. These signs clinically suggested Psoriasis but the biopsy and Reflectance Confocal Microscopy reports suggested otherwise. Although biopsy reported the presence of Munro micro abscess and hyperkeratosis, other characteristic features of psoriasis were not found As the lesions had developed quickly and patient had no prior history of psoriasis or any other cutaneous inflammatory disease, we can argue that this might be an early presentation of psoriasis due to which the biopsy and RCM were negative showing only mild proliferation and inflammation. Also, the failure of remission of the rashes and extensive recurrence of the rash again after few months despite continuous treatment indicates that it is not merely a simple drug reaction. This also suggests that it can be an early presentation of psoriasis.

A very similar case was reported in 1989 [6] which also described psoriasiform eruption triggered by recombinant granulocyte macrophage colony stimulating factor (rGM-CSF) and exacerbated by G-CSF in a patient with breast cancer. The patient however had complete remission of the lesions and no relapse was noted. This is one of the earliest reported cases of G-CSF induced cutaneous eruption. The major difference with our case is that the patient did not have complete remission of her lesions even after 2 months, which makes it fairly arguable whether this case is a drug eruption or early psoriasis. No reports are found stating the induction of the disease Psoriasis itself with G-CSF. 
Later, in 1997 a case was reported stating the worsening of psoriasis after treatment with G-CSF in a patient with small-cell lung cancer. Also, they stated that the withdrawal of G-CSF therapy coincided with the improvement of psoriasis in the patient [7].

However, in later years very few reports of psoriasis like eruption were reported in the literature. Rather other forms of G-CSF induced drug eruptions were reported such as generalized erythematous and indurated papules and plaques with mild epidermal spongiosis and dermal infiltrate of enlarged plump macrophages [8], granulomatous dermatitis [2,9] with enlarged histiocytes clinically manifesting as painful edematous nodules with high fever similar to Sweets syndrome, and cases of G-CSF induced Sweet syndrome reported by Kenneth et al in 1999 and White et al in 2005 [10,11]. It has also been reported that G-CSF stimulates the proliferation of myeloid leukemic cells. Yamashita et al states that although there were no leukemic cells in the peripheral blood or bone marrow, eruptions containing leukemic cells were observed. They explain that those leukemic cells might have responded to hG-CSF and proliferated in the skin [12].

According to a study which analyzed the cytokine profile of this cytokine induced psoriasis like eruption and psoriasis [13], the Polymerase Chain Reaction and immunohistology of G-CSF induced dermatitis resembled psoriasis with regard to epidermal hyperparakeratosis and accumulation of lymphocytes in the upper dermis. This was however different with our patient who showed neutrophil infiltration in the stratum corneum, mild spongiotic changes in the epidermis with perivascular infiltration in the superficial dermis.

High concentrations of G-CSF in the skin is said to induce the production of cytokines by resident cells, including other colony-stimulating factors and interleukins. Activation of macrophage function could stimulate the production of tumor necrosis factor-a, which stimulates keratinocyte-derived monocyte chemotaxis and activating factor. Activated monocytes could further trigger the inflammatory reaction by producing interleukin- 1 and tumor necrosis factor-a like in the pathogenesis of psoriasis which may be the reason for the induction of the psoriatic lesions after its administration.

Although the role of G-CSF in the pathogenesis of psoriasis has not been established, there are reports in which G-CSF could have been related to the induction or exacerbation of psoriasis. In all the cases reported about psoriasis like eruption, the common point is the eruptions of brand-new lesions and worsening

\section{CONCLUSION}

The lesions following G-CSF-induced leukocytosis are characterized by a lymphocytic inflammation and cytokine pattern similar to that detected in active psoriatic lesions and share many of the cellular and molecular changes that are similar to the psoriatic epidermis. Therefore, we speculate that the pattern of skin changes induced by G-CSF, a cytokine involved in the host defense against bacterial infections, is partially overlapping with the induction of cutaneous immune functions that characterize psoriasis and this can be termed as a key pathogenic factor in the induction of psoriasis. Also, the genetic variation of the population can be an important factor for the induction of the psoriasis like eruptions in the patients since not everyone receiving it develops the reaction. Gene polymorphism could be significant to explain the variants in the induction of the disease to G-CSF and should be researched for the better and clearer explanation of this phenomenon. Not many cases have been reported with the association of G-CSF with psoriasis or psoriasis like eruptions. However, the ones, which were found reported in the literature, were also mostly done in the early 90s. So, since the administration of this human recombinant granulocyte colony-stimulating factor has become so common in clinical practice there should be more research and study regarding its adverse cutaneous reactions as they can be life threatening too if not recognized prior to worsening of the patient.

\section{ACKNOWLEDGEMENT}

I would like to thank my professor Dr Song Ji quan and my co-authors for helping me in this paper and also all the colleagues of my department for guiding me and continuously supporting me in my work.

\section{REFERENCES}

1. Fariña MC, Requena L, Dómine M, Soriano ML, Estevez L, Barat A. Histopathology of cutaneous reaction to granulocyte colonystimulating factor: Another pseudomalignancy. J Cutan Pathol. 1998;25:559-62.

2. Ferran M, Gallardo F, Salar A, Iglesias M, Barranco C, Pujol RM. Granulomatous dermatitis with enlarged histiocytes: A characteristic pattern of granulocyte colony-stimulating factor. Report of two cases and review of the literature. Dermatology. 2006;212:188-93. 


\section{www.odermatol.com}

3. Chapman A, El Miedany Y. Psoriasis. Comorbidity Rheum Dis. 2017:81-124.

4. Glass LF, Fotopoulos T, Messina JL. A generalized cutaneous reaction induced by granulocyte colony-stimulating factor. J Am Acad Dermatol. 1996;34:455-9.

5. Yokoyama A, Uemura S, Fujino S, Fujino S, Inoue Y, Kohno N, et al. Eruption caused by recombinant humanG-CSF. Intern Med. 1994;33:641-3.

6. Cho SG, Park YM, Moon H, Kim KM, Bae SS, Kim GB, et al. Psoriasiform eruption trigerred by recombinant granulocytemacrophage colony stimulating factor (rGM-CSF) and exacerbated by granulocyte stimulating factor (rG-CSF) in a patient with breast cancer. Korean Med Sci. 1998;13:685-8.

7. Soutter WP, Mcindoe GA, Hospital H, Robert T, Magnetic S, Unit R. Worsening Psoriasis after treatment with g-csf in a patient with small-cell lung cancer serum basic fibroblast growth factor and vascular endothelial growth factor in metastatic renal cell carcinoma treated with interferon alfa-2b. J Natl Cancer Inst. 1997;89:6-7.

8. Glass LF, Fotopoulos T, Messina JL. A generalized cutaneous reaction induced by granulocyte colony-stimulating factor. J Am Acad Dermatol. 1996;34:455-9.

9. Ozaki S, Funasaka Y, Takubo M, Matayoshi T, Ueno T, Asayama T, et al. Granulocyte colony-stimulating factor-induced granulomatous dermatitis with enlarged histiocytes clinically manifesting as painful edematous nodules with high fever similar to Sweet's syndrome. J Dermatol. 2015;42:414-7.

10. White JML, Mufti GJ, Salisbury JR, Du Vivier AWP. Cutaneous manifestations of granulocyte colony-stimulating factor. Clin Exp Dermatol. 2006;31:206-7.

11. Arbetter KR, Hubbard KW, Markovic SN, Gibson LE, Phyliky RL. Case of granulocyte colony-stimulating factor - induced Sweet's syndrome. Am J Hematol. 1999;61:126-9.

12. Yamashita N, Natsuaki M, Morita H, Kitano Y. Cutaneous eruptions induced by granulocyte colony-stimulating factor in two cases of acute myelogenous leukemia. J Dermatol. 1993;20:473-7.

13. Mössner R, Beckmann I, Hallermann C, Neumann C, Reich K. Granulocyte colony-stimulating-factor-induced psoriasiform dermatitis resembles psoriasis with regard to abnormal cytokine expression and epidermal activation. Exp Dermatol. 2004;13:340-6.

Copyright by Manandhar Upasana, et al. This is an open-access article distributed under the terms of the Creative Commons Attribution License, which permits unrestricted use, distribution, and reproduction in any medium, provided the original author and source are credited.

Source of Support: Nil, Conflict of Interest: None declared. 